\title{
Análisis sobre prácticas de farmacología con animales de laboratorio. Una mirada desde los estudiantes de la Universidad Nacional de San Luis, Argentina
}

Mauricio Roberto Teves ${ }^{\star}$, Farm. ${ }_{1}$, Jésica Daniela Paredes, Bioq. , Emiliano Giraudo, Farm. , María Belén Paredes, Lic. , Mario Franco Moyano, Ph.D ${ }_{1}$, Alejandra Ester Rotelli, Ph.D ${ }_{1}$, Graciela Haydée Wendel, Ph.D ${ }_{1}$, Alejandra Olivia Margarita María, Ph.D ${ }_{1}$, Claudia Patricia Calderón, Ph. $D_{1}$, Lilian Eugenia Pelzer, Ph.D ${ }_{1}$

${ }_{1}$ Farmacología, Departamento de Farmacia, Facultad de Química, Bioquímica y Farmacia, Universidad Nacional de San Luis, San Luis, Argentina

Recibido: 20 de noviembre del 2015 Aprobado: 23 de febrero del 2016

*Autor de correspondencia: Mauricio Roberto Teves, Farmacología, Chacabuco y Pedernera, Universidad Nacional de San Luis, 5700, San Luis, Argentina. Tel: +54 2664520300 interno 6130, fax: +54 266 4431301. Correo electrónico: maurote@unsl.edu.ar

Cómo citar este artículo: Teves Mr, Paredes JD, Giraudo E, Paredes MB, Moyano MF, Rotelli AE, et al. Análisis sobre prácticas de farmacología con animales de laboratorio. Una mirada desde los estudiantes de la Universidad Nacional de San Luis, Argentina. Spei Domus. 2016;12(24). doi: http://dx.doi.org/10.16925/sp.v12i24.1888

Resumen. Introducción: la asignatura Farmacología en Farmacia es de carácter teórico-práctico, dura un cuatrimestre y se imparte en el cuarto año. El desarrollo práctico involucra seis trabajos prácticos de laboratorio (TPL) y cuatro talleres. En tres TPL se realiza experimentación in vivo con animales, en otros dos se trabaja con programas computacionales, y en el restante técnicas in vitro, permitiendo al estudiante valorar el efecto de los fármacos mediante la experimentación, orientado a la investigación biológica y biomédica, además de reconocer la eficacia farmacológica para el tratamiento de enfermedades. Nuestro objetivo fue promover una encuesta para obtener un instrumento orientativo que permita aumentar la calidad de la enseñanza y conocer las percepciones y preferencias sobre las prácticas en cuestión. Metodología: se realizó un estudio transversal y descriptivo, concluido el primer cuatrimestre académico del ciclo lectivo 2015. Resultados: el 90,48 \% aludió que las prácticas con animales resultaron útiles para su formación, y las dos terceras partes consideró necesario implementar alternativas al uso de animales en aspectos relacionados a su educación y formación. El 85,71 \% prefirió las observaciones in vivo; 9,52 \% las in vitro y 4,76\% los programas computacionales. 90,48 $\%$ respondió que volvería a trabajar con animales de laboratorio. Conclusiones: creemos que es pertinente trabajar con animales en esta asignatura, es importante pensar en esta experiencia práctica en la farmacología. Los estudiantes manifiestan su interés por conocer e interpretar los efectos y mecanismos de acción de los fármacos mediante observaciones in vivo o de órganos manipulados por ellos mismos.

Palabras clave: encuesta, estudiantes, farmacia, farmacología, Universidad Nacional de San Luis. 


\title{
Analysis of pharmacology practices with laboratory animals: A look from the perspective of students at the Universidad Nacional de San Luis, Argentina
}

\begin{abstract}
Introduction: The subject Pharmacology in the Pharmacy program is theoretical and practical, lasts a semester, and is taught in the fourth year. Practical development involves six practical laboratory sessions (TPL) and four workshops. Three TPL are for in vivo animal experiments, two for computer programs and one for in vitro techniques, allowing students to evaluate the effect of drugs through experimentation, oriented towards biological and biomedical research, and to recognize pharmacological efficacy for the treatment of diseases. Our objective was to conduct a survey to obtain a guidance instrument that allows to increase teaching quality and to know perceptions and preferences about the practices concerned. Method: A cross-sectional, descriptive study was carried out and concluded in the first four-month period of the academic year 2015. Results: $90.48 \%$ reported that animal practices were useful for training, and two-thirds considered it necessary to implement alternatives to the use of animals in their education and training. 85.71\% preferred in vivo experiments, $9.52 \%$ in vitro observations and $4.76 \%$ computer programs. $90.48 \%$ responded that they would work with laboratory animals again. Conclusions: We believe that it is appropriate to work with animals in this subject; it is important to consider this practical experience in pharmacology. Students expressed their interest in knowing and interpreting the effects and mechanisms of action of drugs through in vivo observations or organs manipulated by them.
\end{abstract}

Keywords: survey, students, pharmacy, pharmacology, Universidad Nacional de San Luis.

\section{Análise sobre práticas de farmacologia com animais de laboratório: uma visão dos estudantes da Universidad Nacional de San Luis, Argentina}

Resumo. Introdução: a disciplina de farmacologia em Farmácia é de caráter teórico-prático, dura um quadrimestre e é dada no quarto ano. O desenvolvimento prático envolve seis trabalhos práticos de laboratório (TPL) e quatro oficinas. Em três TPL, realiza-se experiências in vivo com animais; em outros dois, trabalha-se com programas computacionais e, no restante, técnicas in vitro, o que permite que o estudante avalie o efeito dos fármacos mediante a experiência, orientado à pesquisa biológica e biomédica, além de reconhecer a eficácia farmacológica para o tratamento de doenças. Nosso objetivo foi promover uma enquete para obter um instrumento orientador que permitisse aumentar a qualidade do ensino e conhecer as percepções e as preferências sobre as práticas em questão. Metodologia: realizou-se um estudo transversal e descritivo, concluído o primeiro quadrimestre acadêmico do ciclo letivo 2015. Resultados: 90,48 \% aludiram que as práticas com animais foram úteis para sua formação, e dois terços consideram necessário implantar alternativas ao uso de animais em aspectos relacionados à sua educação e formação. 85,71 \% preferiram as observações in vivo; 9,52 \%, as in vitro, e 4,76 \%, os programas computacionais. 90,48 $\%$ responderam que voltariam a trabalhar com animais de laboratório. Conclusões: acreditamos que é pertinente trabalhar com animais nessa disciplina; é importante pensar nessa experiência prática na farmacologia. Os estudantes manifestam seu interesse em conhecer e interpretar os efeitos e os mecanismos de ação dos fármacos por meio de observações in vivo ou de órgãos manipulados por eles mesmos.

Palavras-chave: enquete, estudantes, farmácia, farmacologia, Universidad Nacional de San Luis. 


\section{Introducción}

La propuesta educativa de la Universidad Nacional de San Luis (UNSL) incluye variados y numerosos programas de pregrado y posgrado cuyo fin obedece principalmente a demandas sociales y a los cambios educativos, científicos y tecnológicos que se generan con la evolución de la sociedad. El ofrecimiento educativo incluye carreras señaladas por el Ministerio de Educación de Argentina como prioritarias, cuyo propósito es la formación de recursos humanos necesarios para el desarrollo del país [1].

La asignatura Farmacología de la carrera de Farmacia se desarrolla en el área de Farmacología y Toxicología del Departamento de Farmacia de la Facultad de Química, Bioquímica y Farmacia de la UNSL; dura un cuatrimestre (de marzo hasta junio), se dicta durante el cuarto año de la carrera, tiene un crédito horario de 120 horas e incluye clases teóricas y actividades prácticas.

En el desarrollo práctico de la asignatura se incluyen diez actividades de asistencia obligatoria que involucran seis trabajos prácticos de laboratorio (TPL) y cuatro talleres. En tres TPL se realiza experimentación in vivo con animales de laboratorio (ratas y ratones). En el primero de ellos se imparten conocimientos sobre el hábitat de los animales, características reproductivas, diferenciación de sexo, modalidades aplicadas para lograr identificaciones individuales temporarias o permanentes -como pinturas en el pelaje, tatuajes, perforación de orejas, entre otros-. Seguidamente, se realiza el adiestramiento de manipulación de animales, el reconocimiento de las distintas vías de administración de fármacos y su práctica en los roedores.

En los siguientes TPL se trabaja con grupos experimentales y grupos de control con el fin de verificar y comparar la acción del fármaco aplicado. Uno de los TPL está orientado a valorar la eficacia y potencia de grupos de fármacos de acción sobre el sistema nervioso central, entre ellos psicofármacos (depresores y estimulantes) e hipnoanalgésicos. El tercer TPL con experimentación in vivo consiste en abordar un modelo experimental generando un edema de pata de rata mediante la administración de carragenina y valorando la acción antiinflamatoria de fármacos mediante el uso de un plestismómetro. Esto se articula con modelos de ratas que han sufrido ulceras gástricas por la administración de AINEs, a las que se efectúa la disección de los estómagos y verificación bajo lupa de la capacidad ulcerativa de los mismos. Esta actividad permite reconocer las reacciones adversas de medicamentos gastrolesivos sobre la mucosa, en este caso, de los propios AINES.

En los dos TPL en los que los alumnos trabajan con programas interactivos computacionales, se busca el reconocimiento de otros modelos con aplicación de nuevas tecnologías. Están orientados a ver efectos de fármacos que actúan sobre la unión neuromuscular y de diferenciación de la respuesta de fármacos adrenérgicos y colinérgicos sobre el sistema cardiovascular.

Por último, el TPL restante se realiza con un baño de órgano aislado utilizando técnicas in vitro, con la finalidad de distinguir los fármacos que afectan el sistema nervioso autónomo y diferenciar la respuesta sobre un mismo órgano, de fármacos con acción sobre el sistema nervioso simpático y parasimpático.

En este caso, los alumnos no tienen contacto con el animal entero. El montaje del órgano es realizado en etapas previas a su ingreso por cuestiones temporales, puesto que es necesario lograr la estabilización del órgano y su aptitud para la experimentación por parte del alumnado.

La duración máxima programada para la realización de los TPL es de tres horas. Previo a cada uno de ellos se efectúa una evaluación escrita referida a los contenidos teóricos vinculados a la actividad práctica. En los TPL que involucran experimentación con animales, los estudiantes tienen la opción de posicionarse frente al aprendizaje desde un rol activo o pasivo. Es decir que no son obligados a la manipulación de los mismos. Toda práctica que incluya manejo de animales por parte del alumnado es supervisada en cada una de sus etapas por el o los docentes responsables del TPL.

Teniendo en cuenta estas prácticas orientadas, se puede inferir que uno de los objetivos de la asignatura es permitir al estudiante valorar el efecto de los fármacos mediante la experimentación, orientándolos a la investigación biológica y biomédica, además de reconocer la eficacia farmacológica para el tratamiento de las enfermedades.

Sibien la carrera de Farmacia de esta Universidad planificó sus actividades y tareas con animales experimentales, advirtió que muchas otras prescindían en sus prácticas de estos modelos, lo cual llevó a considerar las apreciaciones de numerosos organismos que defienden los derechos de los animales, entre ellos 
PETA (People for the Ethical Treatment of Animals), o aquellos que respetan la vida de los animales e impulsan la evitación del dolor y sufrimiento de los mismos, incorporando el término de bioética animal. Desde una perspectiva global, la bioética trata de relacionar juicios de valor con hechos biológicos y en este amplio contexto, los "derechos animales" tienen un claro significado. Hubo quienes realizaron estudios destinados al análisis de actitudes bioéticas en los estudiantes de la carrera de Ciencias Biológicas [2]. Sobre dicha temática, agrupaciones de investigadores trabajaron en la organización de guías para intentar lograr un equilibrio entre los beneficios de la investigación preclínica con la aflicción animal [3].

En el 2014 surgió en Italia una ley de la Delegación Europea en el Decreto Legislativo con respecto a experimentos con animales que incluyó la prohibición de utilizar animales para determinadas investigaciones, como xenotrasplantes, y hasta la cría de perros y gatos, generando en el ámbito de investigación, temores referentes a la obstaculización de avances científicos [4]. En el marco planteado se pueden seguir mencionando numerosos artículos que reflejan la preocupación sobre la utilización de animales y que desde 1994, no solamente manifiestan inquietud por el uso de animales en la experimentación, sino también con respecto a la protección de animales utilizados por sus pieles o finas carnes industrializadas [5].

En nuestro país se encuentra la Asociación Argentina de Especialistas en Animales de Laboratorio (AADEAL) dedicada a la protección de los derechos del animal.

Si bien la filosofía de la farmacología en la carrera de Farmacia de nuestra Universidad es mantener prácticas experimentales con animales - ya que por medio de estos modelos se han logrado avances insuperables en las ciencias médicas-, en búsqueda del equilibrio, se ha impulsado una adecuación a los mencionados requerimientos y en base a la aplicación del principio de las 3 Rs -impulsada por biólogos ingleses por los años 60 que aplicaron las alternativas de reducción, reemplazo y refinamiento al iniciar la búsqueda de un diseño experimental apropiado [6] —, se ha disminuido notoriamente el número de animales utilizados y así también, el número de especies empleadas. En la última década se han reemplazado actividades experimentales con animales por otros modelos de enseñanza. Se establecieron talleres de reconocimiento del uso clínico de fármacos en patologías de mayor prevalencia que son atendidas en la farmacia comunitaria y a su vez, como se ha señalado previamente, se implementaron programas computacionales.

La UNSL cuenta con un bioterio central encargado de la reproducción, cría y mantenimiento de los animales de experimentación (ratas y ratones) La solicitud de animales para la experimentación, tanto aquellas con fines docentes como las realizadas en el marco investigativo, se realiza mediante protocolos que analizados y autorizados por el Comité Institucional de Cuidado y Uso de Animales (Cicua). La presentación de protocolos para trabajos de experimentación y la creación del Cicua fue regulada en sesión ordinaria del honorable Consejo Directivo de la Facultad de Química, Bioquímica y Farmacia de la UNSL mediante Ordenanza No 006/02 [7] y Ordenanza 9/06 [8], utilizando como referencia los documentos Guide for the Care and Use of Laboratory Animals (ILAR) y Guide to the Care and Use of Experimental Animals (CACC) y el documento oficial de Reglamentación ANMAT 6344/96

La percepción de la experimentación animal por parte de la sociedad ha sufrido un cambio importante con el correr del tiempo. Por ello, y en virtud de nuestra práctica pedagógica, planteamos implementar una encuesta a los estudiantes de Farmacología de la carrera de Farmacia, como una herramienta evaluativa de cuyo análisis podríamos obtener un instrumento orientativo que nos permita, no solo aumentar la calidad de la enseñanza, sino también conocer las percepciones y preferencias del alumnado sobre las prácticas en cuestión.

\section{Metodología}

Se realizó un estudio transversal y descriptivo, concluido el primer cuatrimestre académico del ciclo lectivo 2015. Se estableció como criterio de inclusión ser alumno regular y haber aprobado los trabajos prácticos y examinaciones parciales de la asignatura. Se excluyo a aquellos alumnos repitentes del cursado de farmacología y los que se ausentaron a alguno de los seis TPL debido a que en la recuperación de los ausentes al TPL se evalúa solo el contenido teórico del mismo y no la parte práctica. Se le informó a los alumnos que la encuesta no representaba una evaluación académica y que sus respuestas no incidirían en su evaluación final.

Se encuestó a los alumnos seleccionados para conocer su opinión sobre el desarrollo práctico 
llevado a cabo. Se realizó una sencilla encuesta de once preguntas cerradas, con una diferenciación de los encuestados por sexo (masculino y femenino) y según el turno (mañana o tarde) de comisión seleccionado para la realización de los TPL.

La encuesta fue sometida a consideración por cada uno de los docentes integrantes y responsables de los contenidos teóricos y prácticos de la asignatura Farmacología con temas relativos a la carrera de Farmacia de la UNSL.

Las preguntas incorporadas en la encuesta fueron las siguientes:

1. ¿Cree que es ético experimentar con animales?

2. ¿Cree que la experimentación con animales aporta o ha aportado beneficios?

3. ¿Conoce los cuidados que deben darse a los animales de laboratorio?

4. ¿Conoce la procedencia de los animales de laboratorio?

5. ¿Conoce el destino que tendrán los animales después de usados?

6. ¿Conoce la o alguna regulación sobre el uso de animales de laboratorio?

7. ¿Cree que las prácticas con animales son útiles en la formación de un estudiante?

8. ¿Cree necesario el uso de alternativas al uso de animales de laboratorio en su educación y formación?

9. ¿Cuál de las modalidades llevadas a cabo durante el desarrollo de los trabajos prácticos lo/la cautivó más? Enumérelas del 1 al 3 de acuerdo a su preferencia:

\section{Observaciones in vivo Observaciones in vitro Modelos computacionales}

10. ¿Ha quedado satisfecho/a con el uso de animales de experimentación en los TPL?

11. ¿Volvería a trabajar con animales de laboratorio en un futuro?

Todos los registros obtenidos fueron cargados en una base de datos creada en el programa Microsoft Office Excel $2007^{\circ}$, utilizándose la misma para el ulterior análisis.

\section{Resultados}

A partir de 24 alumnos inscritos, se completaron 21 encuestas, 16 de mujeres $(76,19 \%)$ y 5 de hombres $(23,81 \%)$, cuya distribución en base a la comisión de TPL se grafica en la figura 1.

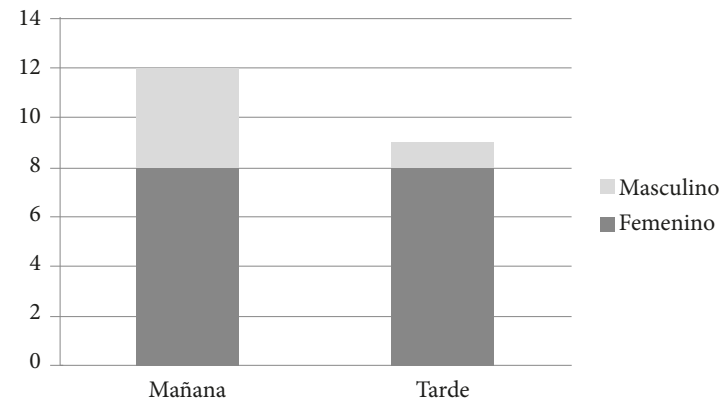

Figura 1. Distribución de alumnos por turno de comisión (mañana o tarde) y sexo (masculino o femenino)

Fuente: elaboración propia

El 76,19\% de los alumnos $(n=16)$ respondió que es ético experimentar con animales, el 19,05 $\%(n=4)$ lo hizo negativamente y $4,76 \%(n=1)$ no sabe/no conoce sobre el cuestionamiento planteado en primera instancia (Figura 2). A su vez, la totalidad de los encuestados coincidió en que la experimentación con animales aporta o ha aportado beneficios para la humanidad.

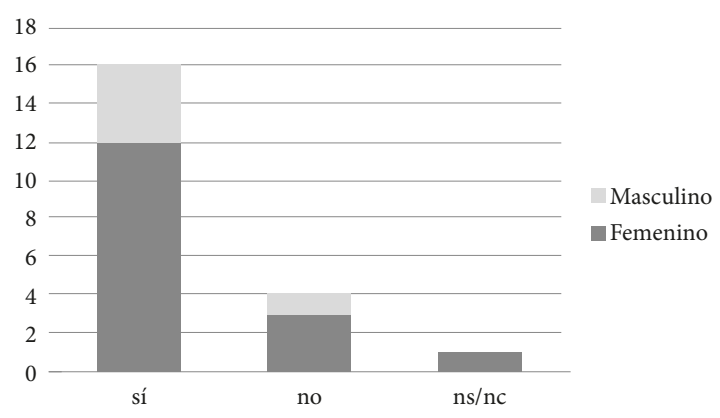

Figura 2. Representación gráfica de las respuestas de los alumnos acerca de si consideran ética la experimentación con animales, diferenciada según el sexo del encuestado (ns/nc: No sabe/No conoce) Fuente: elaboración propia 
El 90,48 \% $(n=19)$ expresó tener conocimiento sobre los cuidados que se le deben brindar a los animales; el 95,24\% $(n=20)$ respondió conocer la procedencia de los animales con los cuales se ha experimentado durante los TPL y el $100 \%$ dijo conocer el destino que tendrán los mismos una vez concluida la experimentación. Estos tres cuestionamientos realizados en la encuesta fueron socializados oportunamente en el primer TPL.

Cuando se indagó acerca del conocimiento del alumnado sobre la existencia de regulación referente a la utilización de animales de laboratorio en la experimentación biológica, un 23,81 \% $(n=5)$ de los encuestados manifestó conocer reglamentación referente a su uso y el 76,19 \% (n=16) indicó lo contrario.

El 90,48 \% ( $n=19)$ aludió a que las prácticas con animales resultaron útiles en su formación, a su vez, las dos terceras partes del alumnado $(n=14$; $66,67 \%)$ consideró necesaria la utilización de alternativas al uso de animales en aspectos relacionados con su educación y formación.

$\mathrm{Al}$ indagar con respecto a la preferencia de las distintas modalidades llevadas a cabo durante los TPL, el 85,71 \% ( $n=18)$ de los alumnos prefirió las observaciones in vivo como primera instancia; 9,52\% $(n=2)$ las observaciones in vitro y solo un alumno (4,76 \%) prefirió los programas computacionales. Además, y en el marco de la misma pregunta, se observó la siguiente distribución de modalidades ordenadas de acuerdo a la preferencia individual (tabla 1).

Los modelos computacionales, señalados como última opción por la gran mayoría, ofrecen variadas ventajas y permiten ensayar con sustancias farmacológicamente activas que se encuentren dentro del arsenal de drogas disponibles del programa, lo cual involucra la principal restricción, señalada oportunamente por los alumnos, al ensayo con sustancias desconocidas o que no estén cargadas en el software.

Por último, al indagar sobre la posibilidad que tuvieron de trabajar activamente en los ejercicios prácticos con animales, el 95,24\% $(n=20)$ contestó afirmativamente. En referencia a si volvería a trabajar con animales de laboratorio en un futuro, 19 encuestados $(90,48 \%)$ se manifestaron positivamente.

Tabla 1. Orden de preferencia de las modalidades para los alumnos (In vivo: Observaciones in vivo; In vitro: Observaciones in vitro; MC: Modelos computacionales)

\begin{tabular}{|l|c|c|c|c|c|c|}
\hline $\begin{array}{c}\text { Orden de } \\
\text { preferencia }\end{array}$ & $\begin{array}{c}\text { Frecuencia } \\
\text { absoluta }\end{array}$ & $\begin{array}{c}\text { Frecuencia } \\
\text { relativa }\end{array}$ & $\begin{array}{c}\text { Porcentaje } \\
(\%)\end{array}$ & $\begin{array}{c}\text { Frecuencia } \\
\text { acumulada }\end{array}$ & $\begin{array}{c}\text { Proporción } \\
\text { acumulada }\end{array}$ & $\begin{array}{c}\text { Porcentaje } \\
\text { acumulado } \\
(\%)\end{array}$ \\
\hline In vivo-in vitro-MC & 17 & 0.81 & 81 & 17 & 0.81 & 81 \\
\hline In vivo-MC-in vitro & 2 & 0.09 & 9 & 19 & 0.90 & 90 \\
\hline In vitro-in vivo-MC & 1 & 0.05 & 5 & 20 & 0.95 & 95 \\
\hline MC-In vitro-in vivo & 1 & 0.05 & 5 & 21 & 1 & 100 \\
\hline
\end{tabular}

Fuente: elaboración propia

\section{Discusión y conclusiones}

Como se mencionó anteriormente, uno de los objetivos de la asignatura es permitir al estudiante valorar el efecto de los fármacos mediante la experimentación, promoviendo con ello una orientación a la investigación biológica y biomédica. Para tal fin, el uso de los animales ha sido fundamental en el desarrollo y avance de dicha investigación. Sin embargo, su uso requiere una justificación válida. Por tal motivo, es que nos planteamos: ¿Es absolutamente necesaria la utilización de animales de experimentación en docencia? ¿Se habrían alcanzado los mismos objetivos si no se hubiesen empleado animales de experimentación? ¿Se encuentra justificada en la docencia la práctica con animales? ¿Puede promoverse una orientación del alumno a la investigación biológica sin el uso de animales? Preguntas formuladas y que nos demuestran la complejidad de esta problemática y la controversia reinante en torno a su utilización.

Algunos autores consideran que las prácticas docentes con animales de laboratorio se hallan en vías de extinción y en algunos casos, se consideran 
obsoletas y son seriamente criticadas [9-11]. Por tal motivo, en nuestra historia de prácticas de laboratorio han quedado en desuso las experiencias con bloqueantes neuromusculares en batracios, entre otras que antiguamente se llevaban a cabo.

En el primer contacto directo con animales de laboratorio entre los estudiantes que asistieron al TPL, se evidenció que los alumnos fueron esquivos frente a su manipulación. Sin embargo, la totalidad de encuestados quedó satisfecha con el uso de animales y un porcentaje mayoritario manifestó la posibilidad de trabajar en un futuro con animales de laboratorio.

Un punto considerado como conflictivo en el análisis de las encuestas surge a partir de la observación de que, las dos terceras partes del total encuestado cree necesaria la aplicación de alternativas al uso de animales en su educación y formación, y pese a ello, mayoritariamente prefirieron la modalidad de las observaciones in vivo, posiblemente debido a que el alumno ya tiene incorporadas las nuevas metodologías de enseñanza y aprendizaje con nuevas tecnologías computacionales o presumiblemente, por el hecho de que las especies que se utilizaron (ratas y ratones) podrían carecer de elevado valor afectivo-emocional por parte del alumnado, algo que solamente se podría revelar con la incorporación de preguntas de tipo conductuales dentro de la encuesta.

Se observó un elevado grado de conocimiento gracias al análisis de consignas dadas a conocer al alumnado durante el desarrollo teórico y práctico, conocimientos referidos al desarrollo preclínico de la experimentación de nuevos fármacos y la trascendencia de los resultados para las pruebas clínicas, cuidado, procedencia y destino de los animales de experimentación.

Otro punto importante a tener en cuenta radica en el elevado porcentaje de encuestados que respondió afirmativamente ante el cuestionamiento de si volverían a trabajar con animales de laboratorio en un futuro. Desde nuestro ámbito de docencia e investigación farmacológica, no podemos desconocer que la historia señala, por ejemplo, que durante los siglos I y II de nuestra era, Galeno ya realizaba vivisecciones a animales y en ocasiones, también el ser humano sirvió como animal de experimentación.

El personal científico ha ido tomando conciencia de que los animales también manifiestan sensibilidad al dolor y capacidad de sufrimiento; así como que todo procedimiento que ocasiona dolor en humanos causa dolor en otras especies de vertebrados [12]

Junto a Russel y Burch y el principio de las 3 Rs [6], en 1964 la Declaración de Helsinki expresó la responsabilidad de la comunidad científica para la humanización de la experimentación animal y la Unión Europea cuenta desde 1986 con la directiva 86/609 [13]. Sin embargo, desde nuestra óptica farmacológica, entendemos que actualmente es difícil pensar en una sustitución absoluta de los animales de experimentación; pero a su vez, resulta importante disminuir el uso de animales, disponer de modelos alternativos, modificar los procedimientos experimentales para evitar o minimizar el dolor y el distress, y aumentar el bienestar de los animales de experimentación. Aunque es deseable sustituir la experimentación científica con animales vivos, su utilización sigue siendo necesaria para proteger la salud humana y animal, así como el medio ambiente [14].

Respecto al objetivo principal de este trabajo de valorar las percepciones y posicionamiento de nuestros alumnos frente a la utilización del animal experimental, su aprobación fue altamente significativa. Sin lugar a dudas, será necesaria la promoción de futuras encuestas a sucesivos estudiantes de la asignatura y evaluar las variaciones temporales de las predilecciones con el fin de alcanzar una adecuación a las exigencias de los estudiantes, que implícitamente son una muestra de aquello que nos demanda la sociedad, apuntando además, la necesidad de profundizar sobre la implicancia de las respuestas con relación a componentes conductuales, cognitivos y afectivos, para lo cual deberíamos incorporar interrogaciones que aborden esos factores, orientados por expertos en el área psicosocial.

\section{Agradecimientos}

Los autores manifiestan su agradecimiento a los estudiantes de Farmacología de la carrera de Farmacia de la Universidad Nacional de San Luis (Argentina) por su participación en el presente estudio.

\section{Referencias}

[1] Pedranzani BE, compilador. La Universidad Nacional de San Luis, en contexto, su historia y su presente. 
$1^{\text {ra }}$ edición. San Luis: Nueva Editorial Universitaria, Universidad Nacional de San Luis, Argentina; 2010.

[2] Fedyk O, Wilczyński KM. Influence of selected factors on development the students' of the biological sciences faculties bioethical attitudes towards experimental animal studies. Psychiatria Danubina. 2015;27(Suppl. 2):S596-S601.

[3] Lindl T, Gross U, Ruhdel I, von Aulock S, Völkel M. Guidance on determining indispensability and balancing potential benefits of animal experiments with costs to the animals with specific consideration of EU directive 2010/63/EU. Altex. 2012;29(2):219-228.

[4] Petrini C. From the Law of European Delegation to the Legislative Decree on experiments with animals: consequences for biomedical research. Clinical Therapeutics. 2014;165(5):e373-5.

[5] Tal H. Animal experimentation, animal welfare and scientific research. Refuat Hapeh Vehashinayim (1993). 2013;30(4):16-22.

[6] Russell WMS, Burch RL. The Principles of Humane Experimental Technique. London: Methuen and Co.; 1959. Disponible en: http://altweb.jhsph.edu/ pubs/books/humane_exp/het-toc

[7] UNSL (Universidad Nacional de San Luis). Ordenanza 006/02. San Luis, Argentina: Honorable Consejo Directivo; 23 de julio de 2002.

[8] UNSL (Universidad Nacional de San Luis). Ordenanza 9/06. San Luis, Argentina: Honorable Consejo Directivo; 3 de abril de 2006 .

[9] Cheong J. The use of animals in medical education: a question of necessity vs. desirability. Theorical Medicine. 1989;10:53-57.
[10] Gruber FP, Dewhurst DG. Alternatives to animal experimentation in Biomedical education. Altex. 2004;21(Suppl. 1):33-48.

[11] Sewell RDE, Stevens RG, Lewis DJA. Pharmacology Experimental Benefits from the use of Computer-Assisted Learning. American Journal of Pharmaceutical Education. 1996;60:303-307.

[12] CіOMs (Council for International Organization of Medica Sciences), ICLAS (The International Council for Laboratory Animal Science). International guiding principles for biomedical research involving animals [internet]. National Institute of Health. December 2012. Disponible en: https://grants.nih.gov/ grants/olaw/Guiding_Principles_2012.pdf

[13] Consejo de la Unión Europea. Directiva 86/609/CEE del Consejo de 24 de noviembre de 1986 relativa a la aproximación de las disposiciones legales, reglamentarias y administrativas de los Estados Miembros respecto a la protección de los animales utilizados para experimentación y otros fines científicos. Diario Oficial de las Comunidades Europeas. 1986 Dic. 18.

[14] Parlamento Europeo, Consejo de la Unión Europea. Directiva 2010/63/Ue del Parlamento Europeo y del Consejo de 22 de septiembre de 2010 relativa a la protección de los animales utilizados para fines científicos. Diario Oficial de las Comunidades Europeas. 2010 Oct. 20 . 